\title{
Prevalencia de Salmonella spp. en carne porcina, plantas de beneficio y expendios del Tolima
}

\author{
Salmonella spp. prevalence in pork, slaughterhouses and \\ butcher's shops in the Tolima department of Colombia
}

\author{
Prevalência de Salmonella spp. em carne suína, \\ plantas de processamento e açougues doTolima
}

\author{
Evelyn C. Arcos - Ávila ${ }^{1}$, Leandro Mora - Cardona ${ }^{1}$, Luz C. Fandiño - de Rubio ${ }^{1,2}$, \\ lang S. Rondón - Barragán 1,2,3
}

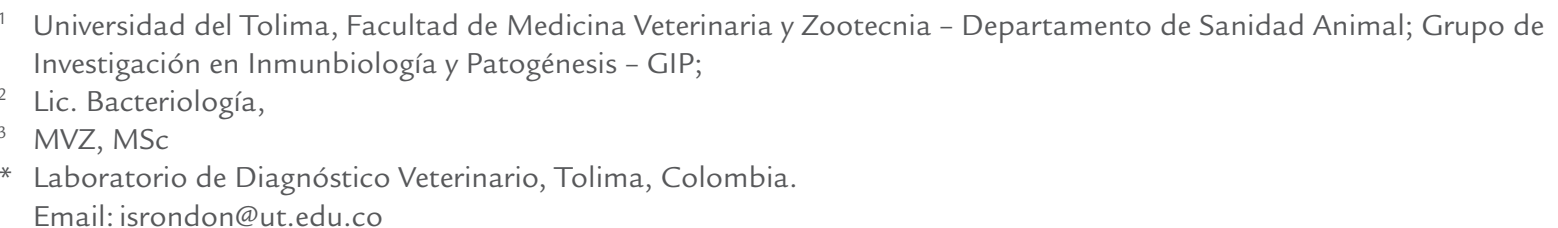

Recibido: julio 05 de $2012 \quad$ Aceptado: abril 01 de 2013

\begin{abstract}
Resumen
La salmonelosis es una enfermedad infectocontagiosa cuya principal manifestación clínica es la gastroenteritis aguda, causada por consumo de agua o alimentos contaminados especialmente carnes. El objetivo del presente trabajo fue determinar de la prevalencia de Salmonella spp. en carne porcina y en ambientes de 6 plantas de beneficio y 14 expendios seleccionados en el departamento del Tolima. Para ello se desarrolló un estudio por conveniencia, seleccionando las plantas por su volumen de sacrificio y el manejo de las buenas prácticas de manufactura. Se obtuvieron muestras $(n=507)$ de canales, ambientes y fómites (i.e. frotis de cuchillos, ganchos, pisos, sifones, mesones y camiones transportadores). Estas muestras fueron sometidas a análisis microbiológico para el aislamiento de Salmonella spp., la cual fue identificada por pruebas bioquímicas y serológicas (Poli A + Vi). Los resultados arrojaron una prevalencia del 4.3\% (25/507) de Salmonella spp. De las 25 muestras positivas $14(56 \%)$ de ellas fueron asiladas de canales y las 11 restantes $(44 \%)$ se aislaron de ambientes; tanto de las plantas de beneficio como de los expendios. En conclusión la presencia de Salmonella spp. en fases de posproducción (beneficio y comercialización) puede presentarse por contaminación con fómites ambientales en el transporte así como en los expendios y reviste importancia dado el riesgo para la salud pública. Este trabajo constituye el primer reporte de prevalencia de Salmonella spp. en plantas de beneficio y expendios en el departamento del Tolima.
\end{abstract}

Palabras clave: plantas de beneficio, porcino, productos cárnicos, Salmonella spp.

\begin{abstract}
Salmonellosis is an infectious disease whose main clinical manifestation is acute gastroenteritis caused by consumption of contaminated water or food especially meat. The aim of this study was to determine the Salmonella spp. prevalence inside
\end{abstract}


pork and the environment from 6 slaughterhouses and 14 butcher's shops all of them selected in Tolima region. A convenience study was realized, choosing slaughterhouses depending on animal reception charge and the good manufacture practices. Sample were obtained $(n=507)$ from environment samples were realized smears on knives, tables, hooks, waste pipes, floors, and transportation trucks. Samples were under microbiologic analysis for Salmonella isolation, which was identified by biochemical and serological test (Poli A +Vi). The results showed a prevalence of Salmonella spp. of 4.93\% (25/507). 14 out 25 positives $(56 \%)$ were isolated from carcass and 11 remaining $(44 \%)$ were isolated from environment; in slaughterhouses as well as butcher's shops. In conclusion, Isolation of Salmonella spp. in post-production phases can be due to cross contamination with environmental fomites in transport as well as in butcher's shops and it's important for the risk in public health. This is the first time, in the Tolima region that the Salmonella prevalence is known in slaughterhouses and butcher's shops.

Key words: abattoirs, swine, meat products, Salmonella spp.

\begin{abstract}
Resumo
A salmonelose é uma doença infecciosa cuja manifestação clínica principal é a gastroenterite aguda causada pelo consumo de água ou alimentos, especialmente carnes contaminados. O objetivo deste estudo foi determinar a prevalência de Salmonellaspp. na carne suína e em ambientes de 6 frigoríficos e 14 açougues selecionados no departamento de Tolima. Foi desenvolvido um por conveniência, a seleção de frigoríficos foi pelo volume de sacrifício e a gestão de boas práticas de manufatura. As amostras foram obtidas $(n=507)$ de carcaças, ambientes e fômites (ou seja, raspagem de facas, ganchos, pisos, sifões mesas e caminhões transportadores). Estas amostras foram submetidas à análise microbiológica para o isolamento de Salmonella spp.,a qual foi identificado por testes bioquímicos e serológicos (Poli A + Vi). Os resultados mostraram uma prevalência de 4,3\% (25/507) de Salmonella spp. Das 25 amostras positivas 14 (56\%) delas foram isoladas de carcaças e os restantes 11 (44\%) foram isolados de ambientes, tanto de frigoríficos como dos açougues. Em conclusão, a presença de Salmonella spp. emfases de pós-produção (transformação e comercialização) pode ocorrer por contaminação por fômites ambientais no transporte e nos pontos de venda e é importante devido ao risco à saúde pública. Este trabalho constitui o primeiro reporte sobre a prevalência de Salmonella spp. em plantas de processamento e açougues, no departamento de Tolima.
\end{abstract}

Palavras chave: plantas de processamento, suínos, produtos carne, Salmonella spp.

\section{Introducción}

La salmonelosis es una de las causas más importantes de gastroenteritis en los humanos, con brotes asociados al consumo de productos de origen porcino, bovino y aviar (Baptista et al., 2009) siendo esta infección la décimo tercera más común en el torrente sanguíneo entre el año 1997-2001; con una ocurrencia de 0.4\% en Norteamérica a 2.3\% en la región Asia-Pacífico (Stephen et al., 2003; Biedenbach et al., 2006). Las infecciones agudas del tracto intestinal están consideradas como una de las más frecuentes en Colombia (Bustos et al., 2008). Desde el punto de vista productivo, genera pérdidas económicas por concepto de muerte, costos de tratamiento y canales de baja calidad; frente a lo cual se han descrito estudios de costo-beneficio que enfatizan sobre la viabilidad de los programas de prevención/control tanto en granjas como en plantas de beneficio (Goldbach y Alban, 2006; Lawson et al., 2009).

La Salmonella es una enterobacteria, gram negativa, en la cual la mayor parte de los serotipos son patógenos para los animales y afectan al hombre accidentalmente (Mastroeni et al., 2000). Pueden provocar infección subclínica, diarrea leve, hasta una severa enfermedad sistémica. La Salmonella infecta cerdos que son reconocidos como una importante fuente de infección para humanos (Mastroeni et al., 2000). En el sistema de producción porcina las infecciones en cerdos están principalmente asociadas con serovares choleraesuis y typhimurium (Mejia, 2003).

La salmonelosis es la toxiinfección alimentaria por zoonosis más importante en países desarrollados. Trevejo et al., (2003) realizaron un estudio de morbimortalidad en los Estados Unidos e informaron que entre 1990 y 1999 se presentaron un total de 11.112 hospitalizaciones, 56.660 casos y 74 muertes atribuidas a infección con Salmonella; de estos pacientes $61 \%$ presentaron gastroenteritis y $23 \%$ septicemia.

En el 2006, 160.649 casos de salmonelosis fueron diagnosticados en la Unión Europea y se considera que de un 10 al 13\% tienen origen en carne porcina (Baptista et al., 2009). En Estados Unidos, se ha descrito que el $56.8 \%$ de la salmonelosis humana puede ser atribuible a los cerdos (BIOHAZ, 2012) y se han llegado a reportar anualmente hasta 1.4 millones de casos ocasionados por Salmonella spp. y se han cuantificado las pérdidas por las mismas cercanas a 25 millones de dólares (Kingsley et al., 2000).

Diversos estudios han demostrado la prevalencia y seroprevalencia de Salmonella en granjas porcinas, así 
como los factores de riesgo asociados a la presencia de la bacteria (Rajić et al., 2004; Henao et al., 2012). De la misma manera, se reporta la prevalencia en carcasas en las plantas de beneficio y en expendios de carne porcina (Arguello et al., 2012; Gonzales-Barron et al., 2012; Methner et al., 2011). En Colombia, Mora et al.,(2003), estudiaron la prevalencia de Salmonella spp. en jugos cárnicos porcinos en plantas de beneficio de Bogotá evaluando 173 muestras de diafragma encontrando un $27.2 \%$ de seropositividad.

El objetivo del presente estudio es determinar la prevalencia de Salmonella spp. en expendios y plantas de beneficio del Departamento del Tolima, en canales porcinas y ambiente incluyendo fómites.

\section{Materiales y métodos}

\section{Población de estudio}

El estudio se llevó a cabo en 6 plantas de beneficio y en 14 expendios de carne porcina en el Departamento del Tolima, se desarrolló un estudio con un criterio de inclusión de las plantas por volumen de sacrificio y el manejo de las buenas prácticas de manufactura (BPM) aprobadas para funcionamiento por el Instituto Nacional de Vigilancia de Medicamentos y Alimentos (INVIMA).Las muestras se recolectaron de canales y ambientes una vez fueron terminadas las labores de beneficio, además se muestrearon los camiones transportadores, y nuevamente las canales cuando llegaron al expendio, en donde también se tomaron muestras de ambientes. Las muestras recolectadas fueron analizadas en el Laboratorio de Diagnóstico Veterinario de la Universidad del Tolima.

\section{Tamaño de la muestra}

Se seleccionaron los municipios del departamento (Chaparral, Fresno, Guamo, Ibagué, Líbano y Mariquita) en donde existía un mayor número de animales sacrificados por semana. Teniendo en cuenta el único estudio realizado en Ibagué por Pabón (1978), en donde la prevalencia de Salmonella fue de $0 \%$ en la planta de beneficio ubicada en este municipio, con un nivel de confianza 95\% y una prevalencia esperada del $5 \%$, se calculó el número mínimo de muestras con la siguiente fórmula descrita por Thrusfield (2007):

$$
n=\frac{z^{2} \times p \times q}{d^{2}}
$$

Dónde:

$$
\begin{array}{ll}
\mathbf{z}^{2}= & \begin{array}{l}
\text { coeficiente del nivel de confianza prefijado }\left(1.96^{2}\right. \\
\text { ya que el nivel de confianza es de } 95 \%)
\end{array} \\
\mathbf{p}= & \text { proporción esperada (en este estudio } 5 \%=0.05) \\
\mathbf{q}= & 1-p \\
\mathbf{d}= & \text { precisión (en este estudio un } 5 \%) \\
n=\frac{(1.96)^{2} \times 0.05 \times(1-0.05)=3.8416 \times 0.05 \times 0.95}{0.025}=72.99(0.05)^{2}
\end{array}
$$

El número mínimo de muestras es de 73. En este estudio se tomaron 507 muestras.

\section{Recolección de muestras}

Para el muestreo en la superficie de las canales se utilizaron los métodos no destructivo y destructivo. El método no destructivo, se realiza sobre la piel de las carcasas con hisopos estériles (3M, USA), los cuales vienen hidratados con agua peptonada, que a su vez sirve para realizar la primera dilución y el pre-enriquecimiento. Se calculó un área de $10 \mathrm{~cm}$ x $10 \mathrm{~cm}$ en cada canal seleccionada se realizaron tres frotis en la mejilla, vientre y muslo respectivamente, de forma vertical (de arriba hacia abajo) y horizontal (derecha a izquierda) (Figura 1).

Se obtuvieron 100 g de carne del vientre y la garganta de cada canal, cortados con hojas de bisturí estériles (método destructivo); estas muestras fueron colocadas en bolsas individuales de cierre hermético.

Las muestras de ambientes fueron recolectadas con esponjas comerciales (3M USA), el cual es un hisopo que contiene agua peptonada tamponada, especial para este tipo de muestreo, realizando frotis con este material sobre cuchillos, mesones, ganchos, sifones, pisos, camiones transportadores (Figura 2). Todas las muestras fueron refrigeradas durante el transporte al laboratorio.

\section{Aislamiento de Salmonella spp}

Para el aislamiento de la Salmonella spp. fueron utilizados los protocolos internacionales estándar (ISO, 2002). Brevemente, las muestras fueron incubadas en agua peptonada-bufferada para su pre-enriquecimiento, con un tiempo de incubación de 24 horas a $37^{\circ} \mathrm{C}$. Las esponjas de referencia EnviroSponge ${ }^{\mathrm{TM}} / \mathrm{HydroS}$ ponge $^{\mathrm{TM}}$, (Biotrace International ${ }^{\circledR}$, Sudáfrica) vienen ya hidratadas con agua peptonada, que a su vez sirve para realizar la primera dilución y el pre-enriquecimiento.

Posteriormente, las muestras fueron dispuestas en caldo tetrationato (Müller-Kauffmann) incubadas a $37^{\circ} \mathrm{C}$ 

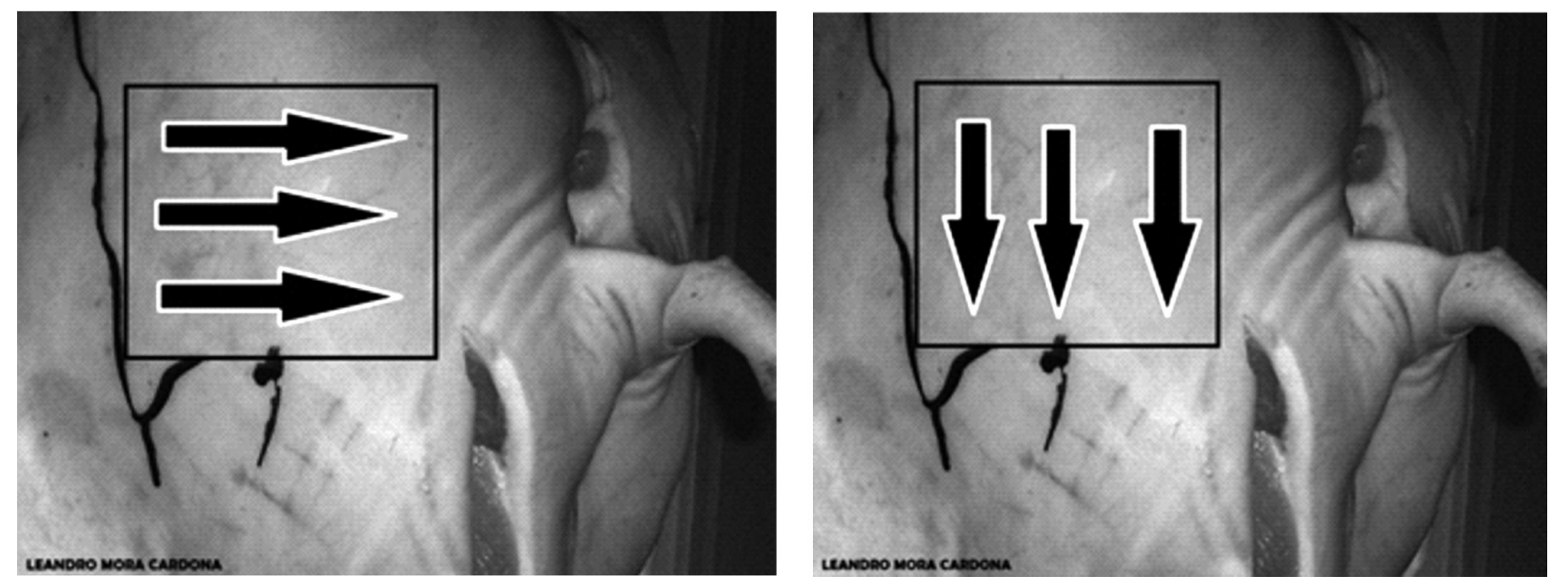

Figura 1. Hisopados realizados en la canal

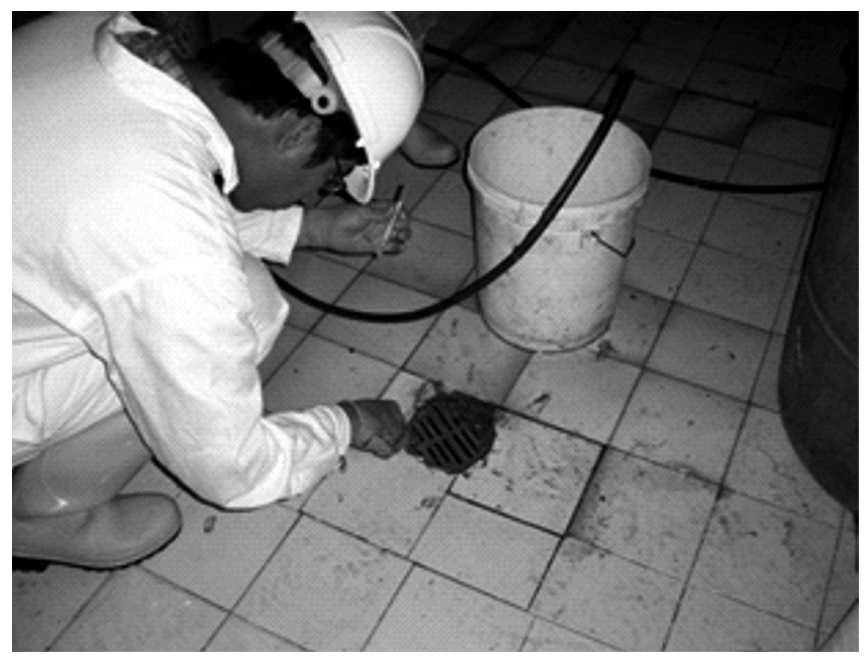

Figura 2. Frotis realizado al sifón. Punto crítico de control.

y Rappaport Vassiliadis (incubadas a $42^{\circ} \mathrm{C}$ ), para su enriquecimiento selectivo. De allí fueron sembradas en agar SS (Salmonella-Shigella, Oxoid, Alemania), agar XLT4 (Xilosa Lisina Tergitol, Oxoid, Alemania) y agar XLD (Xilosa Lisina Desoxicolato, Oxoid, Alemania). Las colonias compatibles fueron subsembradas en agar McConkey y Tripticasa Soya Agar (TSA) confirmadas como Salmonella spp, mediante desafío con anticuerpos Poli A-I + Vi (Difco ${ }^{\circledR} 222641$; USA). De la misma manera fueron confirmadas bioquimícamente mediante galería API $^{\circledR}$ 20E (Biomereux, Francia).

\section{Resultados}

\section{Aislamiento de Salmonella}

De las 507 muestras recolectadas (421 de canal, 86 de ambientes), 25 fueron positivas; arrojando una preva- lencia total de Salmonella spp. del 4.9\%. La prevalencia en canales fue de $3.32 \%(14 / 421)$ y la de ambientes de $12.79 \%$ (11/86). De las 25 muestras positivas 14 (56\%) de ellas fueron aisladas de canales, y las 11 restantes $(44 \%)$ se aislaron de ambientes, tanto de las plantas de beneficio como de los expendios (Tabla 1).

De las muestras positivas obtenidas de las canales, 10 fueron aisladas de la carne $(71.4 \%)$ y 4 del frotis de canal $(28.6 \%)$. Las muestras positivas de ambientes se dividen en 3 aisladas en cuchillos (27.2\%), 3 en ganchos $(27.2 \%), 3$ en sifones $(27.2 \%)$ y 2 en pisos $(18.4 \%)$.

Teniendo en cuenta el total de las muestras positivas observamos que el mayor porcentaje de aislamiento se obtuvo de muestras de carne de la canal (40\%), seguido por los frotis de canal (16\%), los cuchillos $(12 \%)$, ganchos $(12 \%)$, sifón $(12 \%)$ y finalmente los pisos $(8 \%)$. 
Tabla1. Distribución de las muestras positivas $(n=25)$ a Salmonella spp.

\begin{tabular}{|c|c|c|c|}
\hline \multicolumn{3}{|c|}{ Origen } & $\begin{array}{c}\text { Número de } \\
\text { cepas aisladas }\end{array}$ \\
\hline \multirow{6}{*}{ Plantas de beneficio } & \multirow{2}{*}{ Canal } & Carne & 2 \\
\hline & & Hisopado & 1 \\
\hline & \multirow{4}{*}{ Ambientes } & Cuchillo & 3 \\
\hline & & Gancho & 3 \\
\hline & & Pisos & 1 \\
\hline & & Sifón & 1 \\
\hline \multirow{6}{*}{ Expendios } & \multirow{2}{*}{ Canal } & Carne & 10 \\
\hline & & Hisopado & 1 \\
\hline & \multirow{4}{*}{ Ambientes } & Cuchillo & 0 \\
\hline & & Gancho & 0 \\
\hline & & Pisos & 1 \\
\hline & & Sifón & 2 \\
\hline
\end{tabular}

\section{Discusión}

Bacterias tales como Salmonella spp., Aeromonas hydrophylla, Yersinia enterocolitica, Streptococcus suis, Campylobacter coli/jejuni, Listeria monocytogenes y Staphylococcus aureus, son aislamientos comunes de las carcasas, principalmente en cerdos (O'Sullivan et al., 2011). Para el caso de Salmonella spp., se ha considerado que diversos puntos críticos de control están implicados su presencia, los cuales incluyen la recepción de los animales (estabulado), evisceración y faenado de la canal, oreo y refrigeración (Arce et al., 2010; Arguello et al., 2012).

La importancia del estudio de la prevalencia de Salmonella spp. en canales tanto en las plantas de beneficio como en los expendios radica en que muchos de los animales infectados con esta bacteria no evidencian signos clínicos que permitan aislarlos antes de la entrada a la planta de beneficio o no son detectados en la inspección post-mortem (Mainar et al., 2008) y es posible que la contaminación con esta bacteria ocurra con equipos para el procesamiento y sacrificio de los animales así como de los operarios (Mannion et al., 2011; Giovannacci et al., 2011). Es de destacar que para confirmar que la canal está contaminada es necesario aislar la bacteria (Swanenburg et al., 2001).

En el departamento del Tolima, no existen antecedentes que sirvan como punto de referencia de la prevalencia de Salmonella spp. en carne porcina y a nivel nacional son pocos los estudios encaminados a dilucidar la presencia de esta bacteria en la cadena porcina (Mejia, 2007). En un estudio realizado en la planta de beneficio de la ciudad de Ibagué, en el que se obtuvieron muestras de cerdos sacrificados no se hallaron muestras positivas para Salmonella spp. (Pabón, 1978).

La prevalencia de Salmonella spp. en carne de cerdo y ambientes en este estudio fue de $4.9 \%$ - prevalencia en canales de $3.32 \%(14 / 421)$ y la de ambientes de $12.79 \%(11 / 86)$ - en comparación con lo reportado por el laboratorio de salud pública de Bogotá, quienes encontraron una prevalencia de $2.8 \%$ y $0.5 \%$ para los años 2001 y 2002 en muestras de carne animal, no específicamente de cerdo. No obstante, es menor a lo descrito por Mora (2003) quien en un estudio realizado en plantas de beneficio de la ciudad de Bogotá, encontró una prevalencia de Salmonella spp. en jugos cárnicos porcinos de 27.2\%. De la misma manera, Mejia (2007) en plantas de beneficio en la ciudad de Bogotá demostró una prevalencia de 37.82\% (60/156 carcasas)

Los resultados de este trabajo $(3.32 \%$ de prevalencia en carcasas) son cercanos a los reportado para Dinamarca, donde Arguello et al., (2013) reportan un 3.2\% (14/438) de prevalencia en carcasas porcinas; así como a los reportes de Aragaw et al., (2007) quienes reportan una prevalencia de $4 \%$ en carcasas porcinas en Etiopia; sin embargo, difieren de lo reportado en otros países (Tabla 2). Así, Hotes et al., (2010) demos- 
traron una prevalencia de Salmonella de $15.7 \%$ en jugos cárnicos provenientes de carcasas porcinas en Alemania. En Europa, Swanenburg et al., (2001), en un estudio realizado en plantas de beneficio de Holanda, hallaron una prevalencia de Salmonella spp.de un $10 \%$ en carcasas y de $29.4 \%$ de muestras ambientales. Por otro lado, Mürmann et al., (2009) encontraron una prevalencia de Salmonella entérica en salchichas de cerdo de $24.4 \%$ obtenidas de supermercados y expendios en Porto Alegre, Brasil. Prendergast et al., (2010) y Mannion et al., (2012) hallaron una prevalencia del $10.63 \%$ y $5.9 \%$ de Salmonella spp en carcasas porcinas, respectivamente, en plantas de beneficio en Irlanda y Botteldoorn et al., (2003) reportaron una prevalencia de $37 \%$ de Salmonella en carcasas de cerdo en Bélgica. En Canadá, Mainar-Jaime et al., (2008) demostraron en plantas de sacrificio una prevalencia para Salmonella spp. de $17.7 \%$.

Kim et al., (1999), demostraron una relación entre la prevalencia (aislamiento) de Salmonella spp. en las granjas porcinas y el aislamiento de la misma de carcasas; similar a lo reportado por Gebreyes et al., (2004) quienes sustentan que la contaminación de los cerdos con Salmonella empieza cuando los animales dejan el sistema de producción, explicando la contaminación hallada en las plantas de beneficio. Los autores sustentan que las características sanitarias de la granja de origen tienen una fuerte asociación con la positividad en las plantas de beneficio. De igual manera, Berends et al., (1998) demostraron que el 70\% de la contaminación de las carcasas resultaron de los mismos animales siendo portadores y un 30\% proveniente de otros animales portadores y Botteldoorn et al., (2004) demostraron que solo un $25 \%$ de las carcasas fueron contaminadas por genotipos presentes en las heces. No obstante, estudios de Aragaw et al., (2007) demostraron la ausencia de la contaminación de las carcasas a pesar del sacrificio el mismo día de cerdos positivos a Salmonella en muestras de contenido cecal. De otra parte, Berends et al., (1997), describen una asociación estadística nula $(\mathrm{P}>0.10)$ entre la prevalencia de Salmonella en nódulos linfáticos mesentéricos y la contaminación de la carcasa. De la misma manera, Letellier et al., (2009) demostraron que en el $43.4 \%$ de los casos (56 de 129), la serología fue negativa a Salmonella mientras las carcasas fueron positivas, lo cual sugiere una contaminación reciente de los animales durante el

Tabla 2. Prevalencia de Salmonella spp en diferentes fuentes (carcasas, derivados cárnicos y muestras de ambientes de expendios de carne de cerdo) reportada en diferentes países.

\begin{tabular}{|c|c|c|c|}
\hline País & Fuente & Prevalencia $(\%)$ & Referencia \\
\hline Colombia (Tolima) & Carcasa & 3.32 & Arcos et al., (presente estudio) \\
\hline Colombia (Bogotá) & Carcasa & 27.2 & Mora (2003) \\
\hline Colombia (Bogotá) & Carcasa & 37.82 & Mejia (2007) \\
\hline Canadá & Carcasa & 17.7 & Mainar-Jaime et al., (2008) \\
\hline Dinamarca & Carcasa & 3.2 & Arguello et al., (2013) \\
\hline Alemania & Carcasa & 15.7 & Hotes et al., (2010) \\
\hline Bélgica & Carcasa & 37 & Botteldoorn et al., (2003) \\
\hline Irlanda & Carcasa & 10.63 & Prendergast et al., (2010) \\
\hline Irlanda & Carcasa & 5.9 & Mannion et al., (2012) \\
\hline Holanda & Carcasa & 10 & Swanenburg et al., (2001) \\
\hline Etiopia & Carcasa & 4 & Aragaw et al., (2007) \\
\hline Colombia (Tolima) & Ambientes & 12.79 & Arcos et al., (presente estudio) \\
\hline Holanda & Ambientes & 29.4 & Swanenburg et al., (2001) \\
\hline Brasil (Porto Alegre) & Producto procesado (salchicha) & 24.4 & Mürmann et al., (2009) \\
\hline
\end{tabular}


transporte o contaminación cruzada de las carcasas en las plantas de sacrificio.

No obstante, algunos estudios han demostrado una disparidad no solo entre los serotipos aislados entre la planta de beneficio y las granjas sino también en aquellos detectados en la infección (Hurd et al., 2001) lo cual ha sugerido que el transporte y la estabulación de los animales pueden influir en las tasas de infección o aislamiento de la bacteria en las carcasas y ambientes. En el presente estudio solo se evidenciaron dos aislamientos de canal en las plantas de beneficio, sin embargo, fueron 10 los aislamientos al ser muestreado en el expendio, existiendo por tanto posible contaminación en el transporte y el manejo de las canales en los expendios. Vale destacar que las muestras tomadas a los camiones de transporte de las canales fueron negativas a Salmonella.

Otros estudios han sugerido que la contaminación cruzada durante el sacrificio de los animales es importante fuente de contaminación con Salmonella para las carcasas de animales de lotes seronegativos (Swanenburg et al., 2001) y la alta prevalencia de SalmoneIla spp. en las superficies de las carcasas son indicativo de defectos en el equipo o higiene pobre en la línea de beneficio (Käsbohrer et al., 2000).

Así, Mannion et al., (2011) demostraron que al menos el $82 \%$ de los aislamientos de Salmonella spp. en las carcasas son indistinguibles, mediante métodos convencionales y de serotipificación, de aquellos aislados de camiones, estabulados y línea de beneficio así como de las muestras de los operarios, evisceradores y mesones, por lo cual se deduce que puede que estos constituyan una importante fuente de contaminación. Esto concuerda con lo descrito por Giovannacci et al., (2001), quienes a través de genotipificación de macrorestricción resaltan que el ambiente de la planta de beneficio y la contaminación cruzada con cerdos portadores son fuentes importantes de contaminación de las carcasas. En el presente estudio se aisló la bacteria de cuchillos y ganchos los cuales constituyen vehículos o fómites para esta bacteria, por lo cual se debe hacer hincapié en procesos de higiene/limpieza del instrumental utilizado. En el proyecto macro, el análisis de las deficiencias encontradas en plantas de beneficio de porcinos incluyeron fallas en el monitoreo de prácticas de manipulación, falta de rotación de productos de limpieza y desinfección y de separación física de áreas así como deficientes condiciones de infraestructura acorde con la normatividad. De la misma manera, en expendios se halló deficiente manejo de la cadena de frio (Temperaturas y frecuencia), sistemas de esterilización y desinfección de equipos y utensilios y monitoreos de prácticas de higiene. Los vehículos de transporte de la canal y productos cárnicos procesados, en su mayoría son isotérmicos (no poseen equipos de refrigeración).

En la Costa Atlántica Colombiana fueron analizadas 636 muestras de alimento, con el fin de establecer la frecuencia de Salmonella spp., hallando una prevalencia de $7.4 \%$, los resultados obtenidos fueron: carne de res $9.3 \%$, chorizo $12.6 \%$, queso $7.9 \%$, carne de cerdo $5.2 \%$, pollo $1.6 \%$, y arepa de huevo $10.5 \%$; $6.6 \%$ de las muestras positivas eran de alimentos crudos, y $9.7 \%$ eran de alimentos cocidos (Durango et al., 2004). Estos valores difieren de lo evidenciado en el presente estudio.

En el presente estudio la mayor cantidad de cepas fueron aisladas de las carnes en expendios, por lo cual debe considerarse el transporte y la manipulación de la carne como un factor de riesgo asociada a la presencia de la bacteria. Esto aunado al aislamiento de la bacteria de fómites como cuchillos y ganchos que entran en contacto directo con la misma. Prendergast et al., (2009) demostraron una prevalencia de Salmonella spp. en muestras de carne porcina de expendios y supermercados en Irlanda de $2.6 \%$. De la misma manera, demostraron una asociación entre la presencia de Salmonella spp. y el tipo de corte de porcino.

Estudios anteriores han demostrado que las canales porcinas pueden contaminarse con Salmonella spp., a través de la contaminación de equipos o fómites infectados (Manion et al., 2011; Giovannacci et al., 2001). Es de suma importancia recalcar que el mayor porcentaje de fómites en los que se encontró Salmonella fueron cuchillos y ganchos, los cuales entran en contacto directo con las canales afectadas, y son una de las principales causas de contaminación cruzada con esta bacteria. En trabajos de Swanenburg et al., (2001) se describe que el gancho en el cual son suspendidas las canales constituyen un punto crítico en la contaminación de las canales con Salmonella spp.

En sifones y pisos fue posible el aislamiento lo cual reviste importancia pues es allí donde llegan todos los lixiviados de las plantas y de los expendios, por consiguiente se puede conjeturar que en los establecimientos en donde se aisló la bacteria en estos lugares, hay un mayor riesgo que las canales entren en contacto y finalmente se contaminen con la Salmonella spp. Acorde con lo descrito por Swanenburg et al., (2001) quienes atribuyen un papel importante de contaminación de las carcasas a las aguas de drenaje, que pueden diseminar la bacteria. 
Estudios anteriores han aislado Salmonella spp. en ambientes de plantas de beneficio, encontrando una prevalencia de $61 \%$ en sifones, $22 \%$ en separadores de canales, $5 \%$ en manos de los operarios, 3\% en agua del escaldado y $2 \%$ en el equipo de pelado (Swanenburg et al., 2001). En comparación con este estudio encontramos que la prevalencia de Salmonella en sifones es menor $(27 \%)$, la prevalencia de separadores de canales es similar a la prevalencia encontrada en ganchos y cuchillos (27\%) resaltando que estos objetos entran en contacto directo con la canal.

Los factores de riesgo en la contaminación de canales con Enterobacteriaceae aumenta cuando el equipo de pelado no es limpiado y cuando el procedimiento de eviscerado no se hace adecuadamente (Nowak et al., 2007). Esto está correlacionado directamente con la aplicación de buenas prácticas de manufactura en las plantas de beneficio. Aun cuando algunos estudios han descrito la falencia de los protocolos de higiene en la eliminación o control de la presencia de Salmonella, Schmidt et al., (2004) redujeron significativamente la prevalencia de Salmonella spp. en el estabulado de los animales de un rango de $20-100 \%$ a intervalo de 0 a $15 \%$ mediante la aplicación de protocolos de limpieza e higiene estrictos en cerdos asociados. De la misma manera, Mannion et al., (2008) demostraron la necesidad de una limpieza más exigente de los camiones de transporte para reducir el potencial de contaminación de los cerdos.

Es importante concientizar al personal manipulador de carne porcina de emplear las buenas prácticas de manufactura; ya que algunos estudios han demostrado que el número de microorganismos de Salmonella spp. en la superficie de las canales podrían reducirse con procedimientos cuidadosos en el sacrificio como escaldado individual y eliminación cuidadosa del intestino (Nowak et al., 2007).

Se concluye que la prevalencia de Salmonella spp. en carne porcina y ambientes de 6 plantas de beneficio y 14 expendios en el departamento del Tolima fue de $4.9 \%$ en las muestras analizadas (prevalencia en canales de $3.32 \%(14 / 421)$ y la de ambientes de $12.79 \%$ $(11 / 86)$. El mayor aislamiento de bacterias se realizó en los expendios, siendo de importancia la presencia de la bacteria en fómites del ambiente, tales como cuchillos, mesones, que pueden representar una fuente importante de contaminación.

La implementación de planes de limpieza y desinfección de equipos y utensilios utilizados en los procesos de beneficio y desposte porcino; además de la realización de muestreos microbiológicos para verificar que los planes de desinfección cumplen con su objetivo son de vital importancia para reducir el riesgo de contaminación de las canales terminadas con Salmonella spp. Cabe aclarar que las muestras fueron recolectadas de las plantas que estaban en funcionamiento $y$ cumplían con los estándares sanitarios durante las fechas de muestreo.

\section{Agradecimientos}

Al proyecto "Determinación de la prevalencia de Salmonella spp. y cuantificación de factores de riesgo asociados a su presencia en carne porcina del departamento del Tolima".2008W4497-3594 Derivado del convenio 054/2008 CIAT/MADR, financiado por el Ministerio de Agricultura y Desarrollo Rural (MADR) - Asociación Colombiana Porcicultores (ACP). Al Comité Central de Investigaciones y al personal de Laboratorio de Diagnóstico Veterinario con especial interés al auxiliar Luis Hernando Ortiz.

\section{Referencias}

Aragaw K, Molla B, Muckle A, Cole L, Wilkie E, Poppe C, Kleer J, G. Hildebrandt G. The characterization of Salmonella serovars isolated from apparently healthy slaughtered pigs at Addis Ababa abattoir, Ethiopia. Prev Vet Med, 2007; 82: 252-261

Arce M, Avello E, Camacho M, Peña F, Bernal P, Tandrón E. Identificación de riesgos y puntos críticos de control para la implementación de un sistema HACCP en un matadero porcino. REDVET 2010; [Fecha de acceso: 4 de Diciembre de 2011] O3B (11): 1-11. URL: http://www.veterinaria.org/revistas/redvet/n030310B/0310B_DS02.pdf

Argüello H, Carvajal A, Collazos JA, García-Feliz C, Rubio P. Prevalence and serovars of Salmonella enterica on pig carcasses, slaughtered pigs and the environment of four Spanish slaughterhouses. Food Research International, 2012; 45: 905-912.

Arguello H, Sørensen G, Carvajal A, Baggesen DL, Rubio P, Pedersen K. Prevalence, serotypes and resistance patterns of Salmonella in Danish pig production. Res. Vet. Sci. 2013 http:// dx.doi.org/10.1016/j.rvsc.2013.04.001

Baptista F, Alban L, Ersboll A, Nielsen L. Factors affecting persistence of high Salmonella serology in Danish pig herds. Prev Vet Med, 2009; 92: 301-308

Berends BR, van Knapen DA, Mossel SA, Burt S, Snijders JM. Salmonella spp. on pork at cutting plants and the retail level and the influence of particular risk factors. Inter J Food Microbiol, 1998; 44: 207-217.

Berends BR, van Knapen F, Snijders JM, Mossel DA. Identification and quantification of risk factors regarding Salmonella spp. on pork carcasses. Int J Food Microbiol, 1997; 20: 199-206

Biedenbach D, Toleman M, Walsh T, Jones R. Analysis of Salmonella spp. with resistance to extended-spectrum cephalosporins and fluoroquinolones isolated in North America and Latin America: 
report from the SENTRY Antimicrobial Surveillance Program (1997-2004). Diag Microbiol Infect Dis, 2006; 54:13-21.

BIOHAZ. Scientific opinion on an estimation of the public health impact of setting a new target for the reduction of Salmonella in turkeys. EFSA J. 2012; 10: 2616.

Botteldoorn N, Herman L, Rijpens N, Heyndrickx M. Phenotypic and molecular typing of Salmonella strains reveals different contamination sources in two commercial pig slaughterhouses. Applied Environ Microbiol, 2004; 70: 5305 $\square 5314$.

Botteldoorn N, Heyndrickx M, Rijpens N, Grijspeerdt K, Herman L. Salmonella on pig carcasses: Positive pigs and cross contamination in the slaughterhouse. J Applied Microbiol, 2003; 95: 891-903.

Bustos NS, Cortés LF, Domínguez CA, Mendoza LA. 2010. Medición de la carga de enfermedad en una entidad promotora de salud de Colombia año 2008. Bogotá-Colombia. (Tesis Especialización). Colombia. Universidad del Rosario.

Durango J, Arrieta G, Mattar S. Presencia de Salmonella spp. en un área del Caribe Colombiano: un riesgo para la salud pública. Biomédica, 2004; 24: 89-96.

Gebreyes WA, Davies PR, Turkson P-K, Morrow WEM, Funk JA, Altier C, Thakur S. Characterization of antimicrobial-resistant phenotypes and genotypes among Salmonella enterica recovered from pigs on farms, from transport trucks, and from pigs after slaughter. J Food Prot, 2004; 67: 698-705.

Giovannacci I, Queguiner S, Ragimbeau C, Salvat G, Vendeuvre JL, Carlier V, et al. Tracing of Salmonella spp. in two pork slaughter and cutting plants using serotyping and macrorestriction genotyping. J Applied Microbiol, 2001; 90:131S-147.

Goldbach SG, Alban L. A cost-benefit analysis of Salmonella-control strategies in Danish pork production. Prev Vet Med, 2006; 77: $1-14$

Gonzales-Barron UA, Redmond G, Butler F. A risk characterization model of Salmonella Typhimurium in Irish fresh pork sausages. Food Research International 2012; 45: 1184-1193

Henao JS, Ramírez E, Rondón-Barragán I. Análisis de las Buenas Prácticas de Producción en granjas porcícolas del departamento del Tolima y factores de riesgo asociados a la presencia de Salmonella spp. Rev CES Med Zootec, 2012; 7(2): 11-20

Hotes S, Kemper N, Traulsen I, Rave G, Krieter J. Risk factors for Salmonella infection in fattening pigs - An evaluation of blood and meat juices samples. Zoonoses Public Health, 2010; 57:30-38.

Hurd HS, McKean JD, Wesley IV, Karriker LA. The effect of lairage on Salmonella isolation from market swine. J Food Protection, 2001; 64: 939-944

ISO 6579. 2002. International Organization for Standardization. Microbiology - General guidance on methods for the detection of Salmonella. 4th ed. Geneve, Switzerland.

Käsbohrer A, Protz D, Helmuth R, Nöckler K, Blaha T, Conraths FJ, Geue L. Salmonella in slaughter pigs of German origin: An epidemiological study. European J Epidemiol, 2000; 16: 141-146.
Kim JY, Bahnson PB, Troutt HF, Isaacson RE, Weigel RM, Miller GY. 1999. Salmonella prevalence in market weight pigs before and after shipment to slaughter. En: Proceedings of the $3^{\text {rd }}$ International Symposium on the Epidemiology and Control of Salmonella in Pork, Washington, D.C., August 5-7.

Kingsley RA, van Amsterdam K, Kramer N, Bäumler AJ. The shd A gene is restricted to serotypes of Salmonella enterica subspecies I and contributes to efficient and prolonged fecal shedding. Infection Immunity, 2000; 68 (5): 2720-2727.

Lawson L, Jensen J, Christiansen P, Lund M. Cost-effectiveness of Salmonella reduction in Danish abattoirs. Inter J Food Microbiol, 2009; 134:126-132

Letellier A, Beauchamp G, Guévremont S, D'allaire S, Hurnik D, Quessy S. Risk Factors at Slaughter Associated with Presence of Salmonella on Hog Carcasses in Canada. J Food Protection, 2009; 72(11): 2326-2331

Mainar J, Atashparvar N, Chirino M, Rahn K. Survey on Salmonella prevalence in slaughter pigs from Saskatchewan. Can J Vet Res, 2008; 49: 793-796.

Mannion C, Egan J, Lynch P, Fanning S, Leonard N. An investigation into the efficacy of washing trucks following transportation of pigs - A Salmonella perspective. Foodborne Pathogens Disease $2008,5: 261-271$.

Mannion C, Fanning J, McLernon J, Lendrum L, Gutiérrez M, Duggan $\mathrm{S}$, Egan J. The role of transport, lairage and slaughter processes in the dissemination of Salmonella spp. in pigs in Ireland. Food Research International, 2012; 45: 871-879.

Mastroeni P, Chabalgoity J, Dunstan S, Maskell D, Dougan G. Salmonella: Immune Responses and Vaccines. Vet J, 2000; 161:132 164.

Mejía DC. 2007. Aplicación de métodos microbiológicos en planta de sacrificio para la detección de Salmonella spp. en canales porcinas. (Tesis de Pregrado). Colombia. Pontificia Universidad Javeriana. Facultad de Ciencias.

Mejía SW.2003.Epidemiología de la Salmonelosis porcina en granjas de Cataluña y determinación de los factores de riesgo de la infección. (Tesis Doctoral). España. Universidad Autónoma de Barcelona. Departamento de Sanidad y Anatomía animal.

Methner U, Rammler N, Fehlhaber K, Rösler U. Salmonella status of pigs at slaughter - Bacteriological and serological analysis. Int J Food Microbiol, 2011; 151: 15-20

Mora A. 2003. Evaluación de la prevalencia de Salmonella spp en jugos cárnicos de porcinos sacrificados en las plantas de beneficio de Bogotá D.C (Tesis Pregrado). Colombia. Universidad Nacional de Colombia.

Mürmann L, dos Santos MC, Cardoso M. Prevalence, genetic characterization and antimicrobial resistance of Salmonella isolated from fresh pork sausages in Porto Alegre, Brazil. Food Control, 2009; 20:191-195.

Nowak B, von Müffling T, Chaunchom S, Hartung J. Salmonella contamination in pigs at slaughter and on the farm: A field study using an antibody ELISA test and a PCR technique. Int J Food Microbiol, 2007; 115: 259-267. 
O'Sullivan T, Friendship R, Blackwell T, Pearl D, McEwen B, Carman S, Slavić $Đ$, Dewey C. Microbiological identification and analysis of swine tonsils collected from carcasses at slaughter. Can J Vet Res, 2011; 75: 106-111.

Pabón A. 1978. Aislamientos microbiológicos en la planta de beneficio de la ciudad de Ibagué, Tolima. (Tesis de pregrado). Colombia. Universidad del Tolima; Departamento de Sanidad Animal.

Prendergast D, Duggan S, Gonzales U, Fanning S, Butler F, Cormican M, Duffy G. Prevalence, numbers and characteristics of Salmonella spp. on Irish retail pork. Int J Food Microbiol, 2009; 131: 233-239.

Prendergast DM, Grady DO, McCann A, McCabe E, Fanning S, Egan J, Fanning J, Gutierrez M. Application of PCR for rapid detection and serotyping of Salmonella spp. from porcine carcass swabs following enrichment in semi-solid agar, Food Research International 2010; doi:10.1016/j.foodres.2010.08.013 [Fecha de acceso: 3 de diciembre de 2011]. URL: http://www.sciencedirect.com/science/article/pii/S0963996910002942.

Rajić A, McFall M, Deckert AE, Reid-Smith R, Manninen K, Poppe C, Dewey CE, McEwen SA. Antimicrobial resistance of salmonella isolated from finishing swine and the environment of 60 Alberta swine farms. Vet Microbiol, 2004; 104: 189-196.
Schmidt P, O'Connor A, McKean J, Hurd H. The association between cleaning and disinfection of lairage pens and the prevalence of Salmonellaenterica in swine at harvest. Journal of Food Protection, 2004, 67:1384-1388.

Stephen JM, Toleman MA, Walsh TR, Jones RN (2003) SENTRY Program Participants Group. Salmonella bloodstream infections: Report from theSENTRY Antimicrobial Surveillance Program (1997-2001). Int J Antimicrob Agents, 22:395- 405.

Swanenburg M, Van Der Wolf PJ, Snijders J, Van Knapen F. SalmoneIla in slaughter pigs: the effect of logistic slaughter procedures of pigs on the prevalence of Salmonellain pork. Inter J Food Microbiol, 2001; 70: 231-242.

Thrusfield M. 2007. Veterinary epidemiology. 3rd ed. Blackwell Science, Oxford, UK. p. 182-184.

Trevejo RT, Courtney JG, Starr M, Vugia DJ. Epidemiology of salmonellosis in California, 1990-1999: Morbidity, mortality, and hospitalization costs. Am J Epidemiol, 2003; 157:48-57.

Zheng DM, Bonde M, Sørensen JT. Associations between the proportion of Salmonella seropositive slaughter pigs and the presence of herd level risk factors for introduction and transmission of Salmonella in 34 Danish organic, outdoor (non-organic) and indoor finishing-pig farms. Livestock Sci, 2007; 106:89-199 